\title{
ON SCHWARZ'S LEMMA AND INNER FUNCTIONS
}

\author{
BY \\ STEPHEN D. FISHER
}

Introduction. Let $D$ be an arbitrary domain on the Riemann sphere (that is, $D$ is an open connected set on the sphere) and let $p$ be a point of $D$. Let $H^{\infty}(D)$ denote the space of bounded analytic functions on $D$. We consider the problem of maximizing $\left|f^{\prime}(p)\right|$ for $f$ in $H^{\infty}(D)$ with $|f(z)| \leqq 1$ for all $z$ in $D$, and $f(p)=0$. In the case when $D$ is the unit disc $U$ and $p=0$, this is Schwarz's lemma and the solution is classical: the maximum is one and if $\left|f^{\prime}(0)\right|=1$, then $f(z)=e^{i \theta} z$ for some real constant $\theta$. In the general case, the theory of normal families assures us that there is at least one $f_{0}$ in $H^{\infty}(D)$ with $\left\|f_{0}\right\| \leqq 1$ and $f_{0}^{\prime}(p) \geqq\left|f^{\prime}(p)\right|$ for all $f$ in $H^{\infty}(D)$ bounded by 1 which vanish at $p$. We call any such function extremal.

Theorem 1 of this paper provides an elementary proof that there is only one extremal function. We thus add to our problem the question of investigating the properties of the extremal function.

In the case when $D$ is bounded by a finite number of disjoint analytic simple closed curves, the properties of the extremal function have been studied extensively (see [1] and [7]). In this case, the extremal function is known to be analytic over the boundary and to have modulus one there. We show that this is a local property and carries over to all points in the boundary of $D$ where the boundary is sufficiently well behaved, no matter what the remainder of the boundary of $D$ is like. In $\S 1$ we also show that the extremal function is closely related to the problem of removable singularities for bounded analytic functions. In $\$ 2$ we discuss a related extremal problem and show that the properties of its solution imply that on many planar domains $D$, each function analytic and bounded by 1 on $D$ may be approximated uniformly on compact subsets of $D$ by a sequence of inner functions. We also briefly discuss extensions of the results to open Riemann surfaces.

Finally, some remarks on previous work on Schwarz's lemma are in order. Carleson [3, pp. 78-82] and Havinson [8, Theorem 9] have previously shown that the extremal function is unique. Our proof differs substantially from each of theirs and is considerably more elementary. Furthermore, it does not make use of any of the results from the finitely-connected case. Also, our Theorems 2 and 3 and a weaker version of Theorem 4 have previously been proved by Havinson [8, Theorems 22, 28, and 20]; however, as in the case of Theorem 1, the proofs given here are appreciably simpler and more elementary. Indeed, part of the motivation for this paper was to find simpler and more transparent proofs of these results. In particular, it is only in the proof of Theorem 5, which describes the behavior

Received by the editors March 18, 1968 and, in revised form, May 14, 1968. 
of the extremal function at "nice" boundary points, that we make use of any of the results for the finitely-connected case; otherwise the proofs are equally valid for all domains.

\section{Schwarz's lemma.}

THEOREM 1. Let $D$ be an arbitrary domain on the sphere and let $f_{1}$ and $f_{2}$ be two extremal functions for Schwarz's lemma for $D$ and the point $p$ in $D$. Then $f_{1}(z)=f_{2}(z)$ for all $z$ in $D$.

Proof. We may assume with no loss of generality that $D$ supports nonconstant bounded analytic functions. We show that $f_{1}=f_{2}$ by proving that any extremal function is an extreme point of the unit ball of $H^{\infty}(D)$. Since any convex combination of extremal functions is again an extremal function, we will be done.

Suppose, then, that $h \in H^{\infty}(D)$ and $\|f \pm h\|_{\infty} \leqq 1$. Then $|f|^{2}+|h|^{2} \leqq 1$ and hence

$$
|h|^{2} \leqq 1-|f|^{2}=(1-|f|)(1+|f|) \leqq 2(1-|f|) .
$$

Thus, if $g=(1 / 2) h^{2}$, then $g \in H^{\infty}(D)$ and $|g|+|f| \leqq 1$. Hence, to prove that $f$ is extreme, we need only show that if $g \in H^{\infty}(D)$ and $|f|+|g| \leqq 1$ on $D$, then $g \equiv 0$. (This argument reproduces the proof of Lemma 3.1. of [9].)

We may suppose with no loss of generality that $p \neq \infty$. Let $\lambda$ be a complex number of modulus one and consider the function $h=f+\lambda f g$. We have $|h| \leqq|f|+|f||g|$ $\leqq|f|+|g| \leqq 1$. Further, $h^{\prime}(p)=f^{\prime}(p)+\lambda g(p) f^{\prime}(p)$. If $g(p) \neq 0$, then an appropriate choice of $\lambda$ gives $h^{\prime}(p)>f^{\prime}(p)$, a contradiction. Hence, $g(p)=0$.

Now let $g(z)=\sum_{1}^{\infty} a_{j}(z-p)^{j}$ be a power series expansion for $g$ valid in a disc of radius $r$ about the point $p$. If $g \not \equiv 0$, then some $a_{j}$ is not zero. Let $a_{m}$ be the first nonzero coefficient. Consider the function $h(z)=\lambda c g(z) r^{m-1} /(z-p)^{m-1}$ where $\lambda$ is a constant of modulus 1 and $c$ is a small positive number. For $|z-p| \geqq r$ we have $|h| \leqq|g|$. Furthermore, when $c$ is sufficiently small we have $|f|+|h| \leqq 1$ in $|z-p| \leqq r$. Hence, $|f+h| \leqq 1$ on $D$. However, for an appropriate choice of $\lambda$ we have $h^{\prime}(p)$ $=c r^{m-1}\left|a_{m}\right|>0$ and hence $f^{\prime}(p)+h^{\prime}(p)>f^{\prime}(p)$, again a contradiction. Hence, $a_{m}=0$ for all $m$ and thus $g \equiv 0$.

The extremal function for Schwarz's lemma has a number of interesting properties, some of which are developed in the theorems following this useful elementary result.

LEMMA 1. Let $D$ be a domain on the sphere which contains the point at infinity. Let $p$ be a point of $D$ and let $E$ be a closed subset of $\partial D$, the boundary of $D$, with the property that the unbounded component of the complement of $E$ supports nonconstant bounded analytic functions. Let $K$ be a compact subset of $D-\{p\}$ which does not separate $p$ from $E$. Then given $\delta>0$ there is an element $h$ of $H^{\infty}(D)$ with $h(p)>1$, $\|h\|_{K}=\sup _{z \in K}|h(z)| \leqq 1$, and $\|h\|_{D}=\sup _{z \in D}|h(z)| \leqq 1+\delta$.

Proof. Let $D_{1}=\left\{q \in D \mid\right.$ there is an $h \in H^{\infty}(D)$ with $\left.|h(q)|>\|h\|_{K}\right\}$. Since $H^{\infty}(D)$ contains nonconstant functions, $D_{1} \neq \varnothing$. Clearly, $D_{1}$ is open; since $K$ does not 
separate $E$ from $p$ and the unbounded component of the complement of $E$ supports nonconstant bounded analytic functions, $p$ lies in a domain containing points of $D_{1}$. We show that $p$ lies in $D_{1}$ by showing that $D-\left(D_{1} \cup K\right)$ is open. Once it is known that there is at least one function $f$ in $H^{\infty}(D)$ with $f(p)>\|f\|_{K}$, the desired function is found by putting $h=(f+M)\left(M+\|f\|_{K}\right)^{-1}$ for sufficiently large $M$. By an initial elementary transformation we may assume that $p$ lies in the finite plane and $\infty \in D_{1}$.

Let $z_{1} \in D-\left(D_{1} \cup K\right)$. Then $\left|h\left(z_{1}\right)\right| \leqq\|h\|_{K}$ for all $h$ in $H^{\infty}(D)$. Let $A$ be the restriction of $H^{\infty}(D)$ to $K$ so that $A$ is a subalgebra of all the continuous functions on $K$. The multiplicative linear functional $h \rightarrow h\left(z_{1}\right)$ on $A$ is bounded since $z_{1} \in D-D_{1}$ and hence there is a positive measure $m$ on $K$ of mass 1 such that $h\left(z_{1}\right)=\int_{K} h d m$ for all $h$ in $H^{\infty}(D)$. Let $F(z)=\int_{K}\left(\left(\xi-z_{1}\right) /(\xi-z)\right) d m(\xi)$. Then $F$ is analytic off $K$ and $F\left(z_{1}\right)=1$. Hence, $F$ is not zero in a neighborhood $N$ of $z_{1}$. If $z_{2} \in N$, then $F\left(z_{2}\right)=a \neq 0$ and it is easy to check that

$$
h\left(z_{2}\right)=\int_{K} h(\xi) \frac{\left(\xi-z_{1}\right)}{\left(\xi-z_{2}\right)} \frac{1}{a} d m(\xi)
$$

for all $h \in H^{\infty}(D)$. Hence, $\left|h\left(z_{2}\right)\right| \leqq C\|h\|_{K}$ for some constant $C$. (C may depend on $z_{2}$ and $K$.) Thus the functional on $A, h \rightarrow h\left(z_{2}\right)$, is bounded and since it is multiplicative its norm must be 1 . Hence, $\left|h\left(z_{2}\right)\right| \leqq\|h\|_{K}$; thus, each point of $N$ lies in $D-\left(D_{1} \cup K\right)$ and hence $D-\left(D_{1} \cup K\right)$ is open. This completes the proof.

THEOREM 2. Let $D$ be an arbitrary domain on the Riemann sphere with boundary $B$. Suppose $E$ is a compact subset of $B$ such that $E$ is at a positive distance from $B-E$. Let $f$ be the extremal function for Schwarz's lemma for $D$ and the point $p$ in $D$. If $f$ extends to be analytic in the domain bounded by $B-E$, then $E$ is a set of removable singularities for all bounded analytic functions.

Proof. We may assume with no loss that $H^{\infty}(D)$ contains nonconstant functions and that $\infty \in D$.

If the conclusion of the theorem is false, then there are nonconstant bounded analytic functions on $D_{1}$, the unbounded component of the complement of $E$ relative to the sphere $\left[2\right.$, p. 108]. Note that $D \cup[B-E]$ lies in $D_{1}$. Let $f$ be the extremal function for Schwarz's lemma for $D$. By Lemma 1 there is a function $h \in H^{\infty}\left(D_{1}\right)$ such that $h(p)>1$ and $\sup |h(z)| \leqq 1$ for $z \in B-E$. Because $f$ is analytic and bounded by 1 in the domain bounded by $B-E$, $\sup _{z \in E}|f(z)| \leqq 1-\delta$ for some positive $\delta$. Now let $g(z)=((h+M) /(M+1)) f$, where $M$ is a large positive number. Then $g \in H^{\infty}(D), g^{\prime}(p)=\left[f^{\prime}(p)\right](h(p)+M) /(1+M)>f^{\prime}(p)$, and since $|h| \leqq 1$ on $B-E,|g| \leqq 1$ on $D$ when $M$ is sufficiently large. This contradicts the maximality of $f^{\prime}(p)$ and hence $E$ must be a set of removable singularities for all bounded analytic functions.

THEOREM 3. Let $D$ be an arbitrary domain on the sphere and let $f$ be the extremal function for Schwarz's lemma for $D$ and the point $p$ in $D$. If $R$ is the image of $D$ 
under $f$, then either $R$ is the point 0 or $R$ is a domain in the open unit disc $U$ and each bounded analytic function on $R$ may be extended to a bounded analytic function on $U$.

Proof. We may suppose once again that $H^{\infty}(D)$ contains nonconstant functions. Hence, $R$ is a domain in $U$. For $r<1$, let $E_{r}=\{z|| z \mid \leqq r\} \cap[U-R]$, and let $g$ be the extremal function for Schwarz's lemma for $U-E_{r}=D_{r}$ and the point 0 . Then $g(0)=0$. If $g^{\prime}(0)>1$, then $g \circ f \in H^{\infty}(D), g \circ f$ is bounded by one, and $(g \circ f)^{\prime}(p)=g^{\prime}(0) f^{\prime}(p)>f^{\prime}(p)$, a contradiction. Thus $g^{\prime}(0) \leqq 1$. But the identity function $z$ is in $H^{\infty}\left(D_{r}\right)$ and therefore $g(z)=z$. Hence, by Theorem 2, $E_{r}$ is a set of removable singularities for bounded analytic functions. Hence, each function bounded and analytic in $R$ can be extended to be bounded and analytic in $R \cup E_{r}$. Since this is true for all $r<1$, the theorem follows.

Definition. Let $D$ be a domain on the sphere which supports nonconstant bounded analytic functions. If for a point $z$ in $\partial D$ there is an $h$ in $H^{\infty}(D)$ such that $h$ does not extend to be analytic in some neighborhood of $z$, then following [11] we say that $z$ is an essential boundary point for $D$. If each point in $\partial D$ is essential, then we say that $D$ is maximal for $H^{\infty}(D)$.

It is known that each domain $D$ lies in a unique smallest maximal domain $D^{*}$ and each element of $H^{\infty}(D)$ extends to an element of $H^{\infty}\left(D^{*}\right)$ [11, Theorem 11]. Further, two conformally equivalent domains are simultaneously maximal or not [11, Theorem 7].

Proposition 1. Let $D$ be a domain on the sphere and let $D^{*}$ be its maximal domain. Let $f$ be the extremal function for Schwarz's lemma for $D$ and $p$ in $D$ (and hence for $D^{*}$ and $p$ ). Then $f$ is $1-1$ if and only if $D^{*}$ is simply connected.

Proof. If $f$ is $1-1$ in $D$, then $f$ is $1-1$ in $D^{*}$ and hence $f\left(D^{*}\right)$ is maximal. Thus by Theorem 3, $f\left(D^{*}\right)=U$. Hence $D^{*}$ is simply connected.

Conversely, if $D^{*}$ is simply connected, then let $\phi$ be the Riemann map onto $U$ which sends $p$ to 0 . Then $f \circ \phi^{-1}$ is the extremal function for $U$ and the origin so that $f\left(\phi^{-1}(z)\right)=z$ for all $z$ in $U . \therefore f=\phi$ and hence $f$ is $1-1$.

Now we investigate the behavior of the extremal function at the boundary of the domain. We will henceforth assume that $D$ supports nonconstant bounded analytic functions.

THEOREM 4. Let $z_{0}$ be an essential boundary point of $D$ and let $f$ be the extremal function for Schwarz's lemma for $D$ and the point $p$ in $D$. Then $\lim \sup |f(z)|=1$ where $z \in D$ and $z \rightarrow z_{0}$.

Proof. Without any loss of generality $\infty \in D$. Suppose the conclusion of the theorem fails at the point $z_{0}$. Then there is a number $\delta>0$ such that $|f(z)| \leqq 1-\delta$ in $D \cap\left\{\left|z-z_{0}\right|<\delta\right\}$.

Let $N$ be the closed disc of radius $\delta / 2$ centered at $z_{0}$ and let $E=\partial D \cap N$. Since $z_{0}$ is an essential boundary point for $D$, there are nonconstant bounded analytic 
functions on $D_{1}$, the unbounded component of the complement of $E$. Note that $D \subset D_{1}$. Now by Lemma 1 , there is an $h \in H^{\infty}\left(D_{1}\right)$ such that $h(p)>1,|h(z)| \leqq 1$ for all $z$ in $\partial D-\left\{z|| z-z_{0} \mid<\delta\right\}$ and $\|h\| \leqq(1-\delta / 2)^{-1}$. Then $g=h f$ is in $H^{\infty}(D)$, $g^{\prime}(p)=f^{\prime}(p) h(p)>f^{\prime}(p)$, and $\|g\| \leqq 1$, contradicting the maximality of $f^{\prime}(p)$. Therefore, no such $\delta$ exists and the theorem is proved.

Definition. Let $D$ be a domain and $z_{0}$ a point in $\partial D$. We say that $z_{0}$ is free in $\partial D$ if the following is true: there is a conformal map $\phi$ of $D$ which carries $D$ into the upper half-plane in such a manner that the interval $(-a, a)$ lies in $\partial(\phi(D))$ for some $a>0, \lim \phi(z)=0$ for $z \in D, z \rightarrow z_{0}$, and finally, $\{z|| z \mid<\delta\} \cap \partial(\phi(D))$ $=(-\delta, \delta)$ for some $\delta>0$.

For example, if $\gamma$ is a simple closed curve in $\partial D$ which is at a positive distance from $\partial D-\gamma$, then each point of $\gamma$ is free.

THEOREM 5. Let $z_{0}$ be a free point in $\partial D$ and let $f$ be the extremal function for Schwarz's lemma for $D$ and the point $p$ in $D$. Then $f$ extends continuously to $z_{0}$ and $\left|f\left(z_{0}\right)\right|=1$.

We will prove this theorem later, after some weaker, related results which will be used in the proof of Theorem 5 .

We will need the following sharper version of Lemma 1.

LEMMA 2. Let E be a compact subset of the unit circle $\Gamma$ of positive arc-length. Let $K$ be a compact subset of the closed unit disc which does not meet $E$ and let $p$ be a point of $U-K$ which is not separated from $E$ by $K$. Then given $\delta>0$ there is a bounded analytic function $h$ on $U$ which is continuous at each point of $\Gamma-E$ satisfying (1) $|h| \leqq 1$ on $K \cup(\Gamma-E),(2) h(p)>1$ and (3) $\|h\| \leqq 1+\delta$.

Proof. Since $E$ has positive measure there is at least one point $z_{0}$ in $U$ for which the conclusions of the lemma hold.

Let $X=K \cup(\Gamma-E)$ and let $A$ be the set of all functions bounded and analytic on $U$ which are continuous at each point of $\Gamma-E$. Give $A$ the supremum norm and let $\bar{A}$ be the closure of $A$ in this norm. $\bar{A}$ is then a commutative Banach algebra. At this point we use one elementary fact from Banach algebra theory: since the multiplicative linear functional $h \rightarrow h\left(z_{0}\right)$ is not continuous on $A$, the function $\left(z-z_{0}\right)$ is invertible in $\bar{A}$. Now this implies that all rational functions with poles at $z_{0}$ and $\infty$ are in $\bar{A}$ and hence by Runge's theorem, there is an element $g$ in $\bar{A}$ with $|g|<1 / 2$ on $K \cup \Gamma$ and $|g(p)-2|<1 / 2$.

Thus, there is an element $h$ of $A$ with $|h| \leqq 3 / 4$ on $K \cup(\Gamma-E)$ and $h(p)>5 / 4$. The desired function is $(h+M)(1+M)^{-1}$ for sufficiently large $M$.

The author would like to thank Professor T. W. Gamelin for suggesting this proof of Lemma 2.

Proposition 2. Let $D$ be a domain in the upper half-plane. Suppose that $(-1,1)=I$ lies in $\partial D$ and that for each $z$ in I there is an open disc $N=N(z)$ of radius $\delta=\delta(z)$ centered at $z$ such that $N \cap \partial D=(z-\delta, z+\delta)$. Let $f$ be the extremal function for 
Schwarz's lemma for $D$. Then $f$ has nontangential boundary values, $f^{*}$, almost everywhere on $I$ with respect to arc-length and $\left|f^{*}\right|=1$ a.e.

Proof. The existence of the nontangential boundary values is immediate. Suppose $\left|f^{*}\right| \leqq 1-\delta$ on a compact set $E$ in $I$, of positive arc-length. Let $S$ be the square with vertices at $1,-1,1-i$, and $-1-i$. By Lemma 2 there is an $h$ bounded and analytic in the unbounded component of the complement of $S$ with $|h| \leqq 1$ on $\partial D-E, h(p)>1$, and $\|h\| \leqq(1-\delta / 2)^{-1}$. Hence, $g=f h$ is bounded by 1 in $D$ and $g^{\prime}(p)=f^{\prime}(p) h(p)>f^{\prime}(p)$, a contradiction. The proposition follows.

COROLlaRY. If $D$ is bounded by a finite number of disjoint analytic simple closed curves, then the extremal function has modulus one almost everywhere on $\partial D$.

Now we turn to the proof of Theorem 5 and here we make use of a result of Garabedian [7] for the case when $D$ is bounded by a finite number of disjoint analytic simple closed curves. In this case, when $D$ is bounded, Garabedian has shown that there is a function $g$ analytic in $D-\{p\}$ and continuous to $\partial D$ such that near $p, g(z)=(z-p)^{-2}+b_{0}+b_{1}(z-p)+\cdots$, and $\int_{\partial D}|g| d s=2 \pi f^{\prime}(p)$ where $f$ is the extremal function for Schwarz's lemma for $D$ and the point $p$.

Hence,

$$
f^{\prime}(p)=\frac{1}{2 \pi i} \int_{\partial D} g(z) f(z) d z \leqq \frac{1}{2 \pi}\|f\|_{\infty} \int_{\partial D}|g| d s=f^{\prime}(p) .
$$

Thus $(1 / i) g f F \geqq 0$ where $F d s=d z$; further, as Garabedian has shown, both $g$ and $f$ are analytic across $\partial D$.

To prove Theorem 5 it suffices to assume that $D$ is bounded, lies in the upper half-plane, $z_{0}=0,(-a, a)$ lies in $\partial D$ for some $a>0$, and $\{z|| z \mid<a\} \cap \partial D=(-a, a)$. We may also assume that $|p|>a$.

Let $D_{1}, D_{2}, \ldots$ be a sequence of subdomains of $D$ which increase to $D$ satisfying these conditions: (1) $p \in D_{n}$; (2) the boundary of $D_{n}$ consists of a finite number of disjoint piecewise analytic simple closed curves; $(3)(-a, a)$ lies in $\partial D_{n}$ for all $n$, and (4) $\{z|| z \mid<a\} \cap \partial D_{n}=(-a, a)$ for all $n$. We may choose the parametrization of $\partial D_{n}$ so that $d z=d t$ on $(-a, a)$ for each $n$. For each $n$, let $g_{n}$ be the previously described function of Garabedian on $D_{n}$ and let $f_{n}$ be the extremal function for Schwarz's lemma in $D_{n}$ for the point $p$. Clearly $f_{n}$ converges uniformly on compact subsets of $D$ to $f$.

For $0<b \leqq a$ let $D(b)$ be the half disc. $D \cap\{z|| z \mid<b\}$ and let $\Gamma(b)$ be the boundary of $D(b)$.

Now fix $b, 0<b<a$, and for each $n$, let $h_{n}(z)=(z-p)^{2}(z+b)(z-b) g_{n}(z)$. Since $h_{n}$ is continuous to the closure of $D_{n}$ and is analytic in $D_{n}$, for $|z|=b$ and $\operatorname{Im} z>0$, we have

$$
h_{n}(z)=\frac{1}{2 \pi i} \int_{\partial D_{n}} \frac{h_{n}(\xi)}{\xi-z} d \xi
$$


and hence, $\left|h_{n}(z)\right| \leqq C\left[(1 / 2 \pi) \int_{\partial D_{n}}\left|g_{n}\right| d s\right]$ where $C$ is a constant depending on $b$ and on the distance from $p$ to $\partial D_{n}$. Hence, $\left|h_{n}(z)\right| \leqq C_{1}$ for $|z|=b, \operatorname{Im} z>0$, where $C_{1}$ is independent of both $z$ and $n$. Thus $\int_{\Gamma(b)}\left|h_{n}\right| d s \leqq C_{2}$ for all $n$ where $C_{2}$ is a constant. Hence, the $h_{n}$ 's belong to a fixed ball of $H^{1}(D(b))$ and thus there is a subsequence which converges weak-star (as measures on $\Gamma(b)$ ) to a function $h$ in $H^{1}(D(b))$. Thus,

$$
g(z)=\frac{1}{(z-p)^{2}} \frac{1}{(z-b)} \frac{1}{(z+b)} h(z)
$$

is in $H^{1}(D(c))$ for any $c, 0<c<b$. Furthermore, the above estimates show that the $g_{n}$ 's belong to a fixed ball in $H^{1}(D(c))$ and converge uniformly on compact subsets of $D(c)$ to $g$. Finally, since $(1 / i) g_{n} f_{n} \geqq 0$ on $(-c, c)$ for all $n$, and since (some subsequence of) $g_{n} f_{n}$ converges weak-star in $H^{1}(D(c))$ to $g f$ (again, as measures on $\Gamma(c))$, we must have $(1 / i) g f \geqq 0$ on $(-c, c)$. Thus $g f$ is analytic over $(-c, c)$. However, Proposition 2 implies that $|f|=1$ a.e. on $(-c, c)$ so that by a lemma of Rudin [10, Lemma 4.5] both $f$ and $g$ are analytic over $(-c, c)$. Hence, $|f|=1$ everywhere on $(-c, c)$ and this establishes Theorem 5 .

COROLlaRY. If $\gamma$ is a simple closed curve in $\partial D$ at a positive distance from $\partial D-\gamma$, then the extremal function extends continuously to $\gamma,|f|=1$ and $f$ is $1-1$ on $\gamma$. If, in addition, $\gamma$ is analytic, then $f$ extends analytically over $\gamma$.

Proof. The assertions are clear with perhaps the exception of the fact that $f$ is $1-1$ on $\gamma$. To show that we may assume that $\gamma$ is the unit circle, $\Gamma$, and $\partial D-\Gamma$ is at a positive distance from $\Gamma$. We may choose the subdomains $D_{n}$ in the proof of Theorem 5 so that $\Gamma$ is a component of $\partial D_{n}$ for each $n$ and $\partial D_{n}-\Gamma$ is uniformly bounded away from $\Gamma$ for each $n$. The estimates in the proof of Theorem 5 show that the $f_{n}$ 's actually converge uniformly to $f$ on some neighborhood of $\Gamma$; since each $f_{n}$ has modulus one and is $1-1$ on $\Gamma[1$, p. 11], $f$ must be also.

The extremal function will not necessarily extend continuously to points in the boundary which are not free. For example, let $D$ be the domain obtained from the upper half-plane by deleting a sequence of disjoint closed subdiscs $S_{1}, S_{2}, \ldots$ whose centers lie on the positive $y$-axis and decrease to 0 . By the corollary to Theorem 5 , the extremal function $f$ extends analytically over $\partial D-\{0, \infty\}$ and maps each $\partial S_{i}$ $1-1$ onto the unit circle $\Gamma$. Consequently, $f$ cannot be extended continuously to the origin.

For another example, let $D$ be any domain, let $S$ be a straight line interval in $D$, and let $D_{1}=D-S$. Let $f$ be the extremal function for $D_{1}$. It is easy to see by Theorem 2 that $f$ cannot extend continuously to any open interval in $S$. Even more, however, $f$ will not extend continuously to any interior point of $S$. To see this, let $\phi$ be the Riemann map of the sphere minus $S$ onto $U$ and let $D_{2}=\phi\left(D_{1}\right)$. Then $\Gamma \subset \partial D_{2}$ and $\Gamma$ is at a positive distance from $\partial D_{2}-\Gamma$. If $g=f \circ \phi^{-1}$, then $g$ is the extremal function for $D_{2}$ and by the corollary to Theorem $5 \mathrm{~g}$ is $1-1$ and analytic over $\Gamma$. 
The conformal map $\phi^{-1}$ extends continuously to $\Gamma$ and maps $\Gamma$ onto $S$ in such a way that each interior point of $S$ has exactly two inverse images, while each endpoint of $S$ has exactly one. If $p$ is an interior point of $S$ and if $f$ is continuous at $p$, then $g\left(p_{1}\right)=g\left(p_{2}\right)=f(p)$ where $p_{1}$ and $p_{2}$ are the two inverse images of $p$. This contradicts the fact that $g$ is $1-1$.

Finally, we note that if we know something about the boundary of $D$ then we can say more about the range of the extremal function. To this end we say that a nontrivial bounded analytic function $h$ on $D$ is inner if $g=h \circ w$ is an inner function on $U$ where $w$ is the uniformizer of $D$. (That is, $\left|g^{*}\right|=1$ a.e. on $\Gamma$ ). (Note that the definition of inner function is conformally invariant.) A theorem of Frostman [6] asserts that any inner function on $U$ covers the unit disc with the exception of a set of logarithmic capacity zero. Hence, if the domain $D$ has the property that, with the exception of a set of logarithmic capacity zero, each point of $\partial D$ is free, then the extremal function is inner and consequently omits a set of at most zero logarithmic capacity. Similar comments apply if, for example, the Dirichlet problem is solvable in $D$ and the set of nonfree boundary points forms a set of zero harmonic measure.

2. Other extremal problems and approximation by inner functions. As one example of the type of extremal problem that yields easily to the technique of Theorem 1, we discuss Pick-Nevanlinna interpolation.

THEOREM 6. Let $D$ be a domain on the sphere which supports nonconstant bounded analytic functions. Let $z_{0}, z_{1}, \ldots, z_{n}$ be distinct points of $D$ and let $t_{1}, \ldots, t_{n}$ be complex numbers of modulus less than 1 . Let $\Omega=\left\{h \in H^{\infty}(D) \mid\|h\| \leqq 1\right.$ and $h\left(z_{i}\right)=t_{i}$ for $1 \leqq i \leqq n\}$ and let $\Delta=\left\{h\left(z_{0}\right) \mid h \in \Omega\right\}$. If $\Delta$ is nonempty, then it is a closed convex subset of $U$; if $\Delta$ contains more than a single point, then it has a nonempty interior; if $w$ lies in $\partial \Delta$, then there is a unique $f$ in $\Omega$ with $f\left(z_{0}\right)=w$.

Proof. We will suppose throughout that $\Delta$ contains more than one point. Then, as Garabedian has pointed out [7, p. 25] $\Delta$ is convex with respect to circles which have at least one point outside the closed unit disc. Consequently, $\Delta$ has a nonempty interior.

Let $q$ lie in the interior of $\Delta$. The ray $\arg (z-q)=\theta, 0 \leqq \theta<2 \pi$, meets $\partial \Delta$ at exactly one point and every point on $\partial \Delta$ lies in exactly one such ray. Hence, to prove the uniqueness assertion, we need only show that for each $\theta, 0 \leqq \theta<2 \pi$, there is a unique $f \in \Omega$ such that $\arg \left(f\left(z_{0}\right)-q\right)=\theta$ and $\left|f\left(z_{0}\right)-q\right|=\sup \left\{\left|h\left(z_{0}\right)-q\right| \mid h \in \Omega\right.$ and $\left.\arg \left(h\left(z_{0}\right)-q\right)=\theta\right\}$. Clearly there is at least one such function; if $f_{1}$ is another, then so is $\frac{1}{2} f+\frac{1}{2} f_{1}$. Consequently, we need only show that such an $f$ is an extreme point of the unit ball of $H^{\infty}(D)$.

Suppose, then, that $g \in H^{\infty}(D)$ and $|f|+|g| \leqq 1$ in $D$. We may assume with no loss of generality that $g\left(z_{i}\right)=0$ for $1 \leqq i \leqq n$. Let $h=f+\lambda c g(f-q)$ where $|\lambda|=1$ and $c$ is a small positive number. We have $|h| \leqq|f|+c|g||f-q| \leqq|f|+|g| \leqq 1$ when $c$ 
is sufficiently small. Hence, $h \in \Omega$. Further, if $g\left(z_{0}\right) \neq 0$, then $h\left(z_{0}\right)-q=\left(f\left(z_{0}\right)-q\right)$ $\left(1+c \lambda g\left(z_{0}\right)\right)$ and hence for an appropriate choice of $\lambda$, $\arg \left(h\left(z_{0}\right)-q\right)=\theta$ and

$$
\left|h\left(z_{0}\right)-q\right|=\left|f\left(z_{0}\right)-q\right||1+c| g\left(z_{0}\right)||>\left|f\left(z_{0}\right)-q\right|,
$$

a contradiction. Hence, $g\left(z_{0}\right)=0$. In a similar fashion, $g^{(K)}\left(z_{0}\right)=0$ for $K=1,2, \ldots$ and hence $g \equiv 0$, as desired.

If $\Delta$ consists of a single point, then the above proof is easily modified to show that $\Omega$ consists of precisely one function.

In the case when $D$ is bounded by a finite number of disjoint analytic simple closed curves, Garabedian [7] has exhaustively treated the properties of the extremal function. In the general case, the extremal function may be used to prove an interesting approximation theorem. We first need this sharper version of Lemma 2.

LEMMA 3. Let $E$ be a closed subset of the unit circle $\Gamma$ of positive arc-length. Let $K$ be a compact subset of the closed unit disc which does not meet $E$ and let $z_{0}, z_{1}, \ldots, z_{n}$ be distinct points of $U$ which are not separated from $E$ by $K$. Then there is a bounded analytic function $g$ in $U$ which is continuous at each point of $\Gamma-E$ such that $g\left(z_{i}\right)=1$ for $1 \leqq i \leqq n, g\left(z_{0}\right)=2$, and $|g| \leqq 1$ on $K$ and on $\Gamma-E$.

Proof. The proof is a simple extension of the proof of Lemma 2 together with an elementary convexity argument. For this reason, we omit it.

The next theorem is an example of the type of approximation theorem that we can prove from Theorem 6 and Lemma 3. We purposely keep the hypotheses strong to simplify the proof.

THEOREM 7. Let $D$ be a domain on the sphere whose boundary is the union of countably many disjoint nontrivial continua $\gamma_{1}, \gamma_{2}, \ldots$ (so that the Dirichlet problem may be solved in D) where each $\gamma_{i}$ is either a simple closed curve or an analytic arc. Suppose further that the set of accumulation points of the $\gamma_{i}$ has harmonic measure zero. Then each function analytic and bounded by 1 on $D$ is the pointwise limit in $D$ of a sequence of inner functions.

Proof. Let $z_{1}, z_{2}, \ldots$ be a sequence of distinct points of $D$ which accumulate at $z_{0} \in D, z_{0} \neq z_{i}$ for $i=1,2, \ldots$ We may assume that no $z_{i}$ is the point at $\infty$. Let $h \in H^{\infty}(D)$ with $\|h\| \leqq 1$ and put $\Omega_{n}=\left\{g \in H^{\infty}(D) \mid\|g\| \leqq 1\right.$ and $g\left(z_{i}\right)=h\left(z_{i}\right)$, $1 \leqq i \leqq n\}$. If we select one $h_{n}$ from each $\Omega_{n}$, then it is clear that $h_{n} \rightarrow h$ uniformly on compact subsets of $D$. We need only show that the $h_{n}$ 's may be chosen to be inner functions.

Since $\Omega_{n}$ is not empty, the set $\Delta_{n}=\left\{g\left(z_{0}\right) \mid g \in \Omega_{n}\right\}$ is not empty. If $\Delta_{n}$ has more than one point, then by Theorem 6 there is a unique $h_{n} \in \Omega_{n}$ with $\arg h_{n}\left(z_{0}\right)=\theta$ (for some real $\theta, 0 \leqq \theta<2 \pi$ ) and $\left|h_{n}\left(z_{0}\right)\right| \geqq\left|g\left(z_{0}\right)\right|$ for all $g \in \Omega_{n}$ with $\arg g\left(z_{0}\right)=\theta$. If $\Delta_{n}$ has only one point, then by Theorem $6, \Omega_{n}$ has only one function, which we call $h_{n}$. In either case we contend that $h_{n}$ is an inner function. 
To see this, suppose the contrary. Then there is a set $F$ in the unit circle $\Gamma$ of positive arc-length with $\left|\left(h_{n} \circ w\right)^{*}\right| \leqq 1-\delta$ on $F$, where $\delta>0$ and $w$ is the uniformizer of $D$. $w$ has boundary-values a.e. on $F$ and these values lie in $\partial D$ and form a set $E$ of positive harmonic measure. Since $\partial D=\bigcup \gamma_{i}$, there is an $i$ such that $E_{\imath}=E \cap \gamma_{i}$ has positive harmonic measure; we may assume that each point of $E_{i}$ has a neighborhood which meets $\partial D$ in just $\gamma_{i}$. To simplify notation let $E=E_{i}$.

Let $\phi$ be a conformal map of the component of the complement of $\gamma_{i}$ that contains $D$ onto the open unit disc and let $D_{1}=\phi(D)$. If $\psi=\phi^{-1}$, then $\psi$ extends continuously to the closed unit disc and maps $\Gamma$ into $\gamma_{i}$. Furthermore, $\psi$ carries harmonic measure for $D_{1}$ to harmonic measure for $D$. Let $E_{1}$ be the set in $\Gamma$ corresponding to $E$ in $\gamma_{i}$; hence, $E_{1}$ has positive arc-length. Further, since $\psi$ is continuous to $\Gamma$, each point of $E_{1}$ has a neighborhood which meets $\partial D_{1}$ in just $\Gamma$.

If we put $H_{n}=h_{n} \circ \psi$, then $H_{n}$ is the unique function solving the extremal problem

$$
\sup \left\{\left|g\left(\phi\left(z_{0}\right)\right)\right| \mid g \in H^{\infty}\left(D_{1}\right),\|g\| \leqq 1, g\left(\phi\left(z_{i}\right)\right)=h\left(z_{i}\right)\right.
$$

$$
\text { for } \left.1 \leqq i \leqq n \text {, and } \arg g\left(\phi\left(z_{0}\right)\right)=\theta\right\} ;
$$

(or, in the case when $\Omega_{n}$ has only one element, $H_{n}$ is the only function analytic and bounded by 1 on $D_{1}$ with $g\left(\phi\left(z_{i}\right)\right)=h\left(z_{i}\right)$ for $1 \leqq i \leqq n$.) By Lemma 3 the boundary values of $H_{n}$ must have modulus 1 a.e. on $E_{1}$. However, at almost all the points of $F, \phi \circ w$ has a radial limit; since $H_{n}\left(\phi\left(w\left(r e^{i \theta}\right)\right)\right)=h_{n}\left(w\left(r e^{i \theta}\right)\right)$ this implies that the boundary value of $h_{n} \circ w$ at $e^{i \theta}$ must be equal to the boundary value of $H_{n}$ at the corresponding point of $E_{1}$ (by the sectorial limit theorem, for example). This shows that the boundary values of $h_{n} \circ w$ cannot have modulus less than one on $F$. Hence, $h_{n} \circ w$ must be inner.

COROLlaRY. Suppose that $\partial D$ is the union of countably many disjoint analytic simple closed curves $\gamma_{1}, \gamma_{2}, \ldots$ with the property that the set of accumulation points of the $\gamma_{i}$ has harmonic measure zero. Let $h \in H^{\infty}(D)$ with $\|h\| \leqq 1$. Then there is a sequence $\left\{h_{n}\right\}$ of elements of $H^{\infty}(D)$ such that the boundary values of each $h_{n}$ on $\partial D$ have modulus one almost everywhere with respect to harmonic measure and $h_{n} \rightarrow h$ uniformly on compact subsets of $D$.

This corollary is known for the case when $\partial D$ consists of finitely many disjoint analytic simple closed curves; in particular, it follows from Garabedian's solution of the Pick-Nevanlinna interpolation problem.

In a fashion similar to Theorem 7 we can prove

THEOREM 8. Suppose that $D$ is a domain on the sphere with $\partial D=E \cup \bigcup_{i=1}^{\infty} \gamma_{i}$ where each $\gamma_{i}$ is a nontrivial continuum, $\gamma_{i}$ is at a positive distance from $\partial D-\gamma_{i}$ for each $i$, and $E$, which is the set of accumulation points of the $\gamma_{i}$, is disjoint from each $\gamma_{i}$ and has logarithmic capacity zero. Then each function analytic and bounded by 1 on $D$ is the pointwise limit on $D$ of a sequence of inner functions. 
The pointwise density of the inner functions in the unit ball of $H^{\infty}(U)$ has been successfully exploited by Forelli in [5] to discover the isometries of $H^{p}(U)$. (See [4] or [5] for the relevant definitions.) His proof carries over to all finitely-connected domains and some infinitely-connected ones. For example, let $D$ be obtained by deleting from the open unit disc $U$ a sequence of pairwise disjoint closed subdiscs centered at the points $1-1 / n^{2}$. It is known in this case that $H^{\infty}(D)$ is dense in $H^{p}(D)$ (cf. [4]). Let $t_{0}$ be the point for which the $H^{p}(D)$ norm is determined, $1 \leqq p<\infty$, and let $\mu$ be harmonic measure on $\partial D$ for $t_{0}$. If $T$ is a linear isometry of $H^{p}(D)$ into $H^{p}(D), 1 \leqq p<\infty$, then $T(f)=F \cdot f(\psi)$ where $\psi$ is an analytic function which maps $D$ into $D$, (the boundary value function of) $\psi$ maps $\partial D$ onto almost all $(d \mu) \partial D$, and $F \in H^{p}(D)$. Further, $F$ is related to $\psi$ by $\int_{X}|F|^{p} d \mu=\int_{X} 1 / p(\psi) d \mu$ where $p d \mu$ is harmonic measure for the point $\psi\left(t_{0}\right)$, and $X=Y \Delta Z$ where $Z$ is a $\mu$-null set and $Y=\psi^{-1}(W)$ where $W$ is $\mu$-measurable.

There are a number of other extremal problems whose solutions may easily be proved to be unique (up to rotation) by the technique of Theorem 1. Among them are

(1) Maximize $|f(q)|$ over all $f \in H^{\infty}(D)$ which are bounded by 1 and which vanish at $p \in D-\{q\}$, or vanish at points $p_{1}, p_{2}, \ldots$ provided that there is some nontrivial $h \in H^{\infty}(D)$ which vanishes at all the $p_{i}$.

(2) Maximize $\left|f^{\prime}(p)\right|$ over all $f \in H^{\infty}(D)$ bounded by 1 on $D$ which do not vanish in $D$, where $D$ supports nonconstant bounded analytic functions.

(3) Let $D$ be a domain which supports nonconstant bounded analytic functions. The extremal problem is to maximize $|f(p)-f(q)|$ over all $f \in H^{\infty}(D)$ with $\|f\| \leqq 1$ where $p$ and $q$ are distinct points of $D$.

(4) This is a generalization of (3). Let $D$ be a domain which supports nonconstant bounded analytic functions, $K$ a compact subset of $D$ with no interior which does not separate the plane. Let $\mu$ be any nonzero measure on $K$. The extremal problem is to maximize $\left|\int_{K} f d \mu\right|$ over all $f \in H^{\infty}(D)$ with $\|f\| \leqq 1$. (If $\mu \geqq 0$, then we can make the problem more interesting by requiring that $f$ vanish at some point of $D-K$.)

Concerning extensions of the results of this paper to open Riemann surfaces we will content ourselves with the following: suppose that $D$ is an open subset of some compact Riemann surface $S$ such that $S-D$ has interior; then Theorem 1 is true. (All derivatives are taken with respect to a fixed system of coordinates centered at $p$. Of course, $H^{\infty}(D)$ is nontrivial in this case.) Since $S-D$ has interior, there is a function $h \in H^{\infty}(D)$ such that $h$ has a zero of order one at $p \in D, h$ does not vanish on $D-\{p\}$, and $|h| \geqq \delta>0$ off some small neighborhood of $p$. To prove Theorem 1 we proceed exactly as before to the point where $g$ is divided by $(z-p)^{m-1}$; here we divide instead by $h^{m-1}$.

\section{REFERENCES}

1. L. Ahlfors, Bounded analytic functions, Duke Math. J. 14 (1947), 1-11.

2. L. Ahlfors and A. Beurling, Conformal invariants and function-theoretic null sets, Acta Math. 83 (1950), 101-129. 
3. L. Carleson, Selected problems on exceptional sets, Van Nostrand, Princeton, N. J., 1967.

4. S. Fisher, Rational functions, $H^{\infty}$, and $H^{p}$ on infinitely-connected domains, Illinois J. Math. 12 (1968), 513-523.

5. F. Forelli, The isometries of $H^{p}$, Canad. J. Math. 16 (1964), 721-728.

6. O. Frostman, Potentiel d'équilibre et capacité des ensembles avec quelques applications à la théorie des fonctions, Medd. Lunds Univ. Mat. Sem. 3 (1935), 1-118.

7. P. R. Garabedian, Schwarz's lemma and the Szegö kernel function, Trans. Amer. Math. Soc. 67 (1949), 1-35.

8. S. Ya. Havinson, Analytic capacity of sets, joint nontriviality of various classes of analytic functions and the Schwarz lemma in arbitrary domains, Amer. Math. Soc. Transl. (2) 43 (1964), 215-266.

9. R. R. Phelps, Extreme positive operators and homomorphisms, Trans. Amer. Math. Soc. 108 (1963), 265-274.

10. W. Rudin, Analytic functions of the class $H^{p}$, Trans. Amer. Math. Soc. 78 (1955), 44-66.

11. - Some theorems on bounded analytic functions, Trans. Amer. Math. Soc. 78 (1955), $333-342$.

MASSACHUSETTS Institute OF TEChNOLOGy,

Cambridge, MassachusetTs 